\title{
On the Resolution of an Inverse Problem by Shape Optimization Techniques
}

\author{
Chahnaz Zakia Timimoun (D) \\ Département de Mathématiques, Faculté des Sciences Exactes et Appliquées, \\ Laboratoire de Mathématique et ses Applications, Université Oran1 Ahmed Ben Bella, Oran, Algeria
}

Correspondence should be addressed to Chahnaz Zakia Timimoun; c.timimoun@yahoo.fr

Received 26 November 2018; Revised 25 February 2019; Accepted 26 March 2019; Published 15 April 2019

Academic Editor: Changbum Chun

Copyright (C) 2019 Chahnaz Zakia Timimoun. This is an open access article distributed under the Creative Commons Attribution License, which permits unrestricted use, distribution, and reproduction in any medium, provided the original work is properly cited.

In this work, we want to detect the shape and the location of an inclusion $\omega$ via some boundary measurement on $\partial \Omega$. In practice, the body $\omega$ is immersed in a fluid flowing in a greater domain $\Omega$ and governed by the Stokes equations. We study the inverse problem of reconstructing $\omega$ using shape optimization methods by defining the Kohn-Vogelius cost functional. We aim to study the inverse problem with Neumann and mixed boundary conditions.

\section{Introduction}

The problem of detecting an inclusion $\omega$ immersed in a fluid flowing in a greater bounded domain $\Omega$ has been researched by many authors. In [1], Alvarez et al. investigated this problem to find the location and the shape of $\omega$ using the measurement of the velocity of the fluid and the Cauchy forces on the boundary $\partial \Omega$. Badra et al. [2] studied the same problem using the least-squares functional and Caubet et al. in [3] solved the problem using the Kohn-Vogelius functional with Dirichlet boundary conditions.

In this work we assume that the fluid is governed by Stationary Stokes equations with homogeneous Neumann boundary condition on the interior boundary and nonhomogeneous Dirichlet boundary condition on the exterior boundary. We solve our inverse problem by minimizing the Kohn-Vogelius cost functional. Then we characterize the gradient of this functional.

The paper is organized as follows: in the first part of the paper, we introduce the notations and the overdetermined problem that we consider. In the second part we state the main results of this work and we compute the first order derivative of the cost functional.

In order to do so, we need to fix some notation and definitions. For a bounded Lipschitz open subset $\Omega \subset \mathbb{R}^{d}$ $(d=2$ or 3$)$ with a smooth boundary $\partial \Omega$, $\mathbf{n}$ represents the external unit normal to $\partial \Omega$, and for a smooth enough function $u$, we note, respectively, $\partial_{n} u$ and $\partial_{n n}^{2} u$, the normal derivative and the second normal derivative of $u$. Recall that $\partial_{n} u:=\nabla u n$. The tangential differential operators which will be noted by the subscript $\Gamma$ are defined on $\partial \Omega$ as follows:

$$
\nabla_{\Gamma} w:=\nabla w-(\nabla w n) \otimes n \quad \forall w \in \mathbf{W}^{1,1}(\partial \Omega)
$$

where $\otimes$ denotes the tensor product. For more details on tangential differential operators, we refer to [4, Section 5.4.3].

Finally, for a nonempty open subset $O$ of $\partial \Omega$, we recall that

$$
H_{00}^{1 / 2}(O):=\left\{u_{\mid \mathrm{O}}, u \in H^{1 / 2}(\partial \Omega), u_{|\partial \Omega| \bar{O}}=0\right\} .
$$

\section{The Problem Setting}

Let $\Omega$ be a bounded, connected and Lipschitz open subset of $\mathbb{R}^{d}(d=2$ or 3$)$. Given $\delta>0$, consider $\mathcal{O}_{\delta}$ as the set of admissible geometries such that

$$
\begin{aligned}
& \mathcal{O}_{\delta} \\
& \quad:=\left\{\omega \subset \Omega \text { be an open set with a } C^{2,1} \text { boundary such that } d(x, \partial \Omega)\right. \\
& >\delta \forall x \in \omega\} .
\end{aligned}
$$


Take now $\Omega_{\delta}$ as an open set with a $C^{\infty}$ boundary and satisfy the following assumption:

$$
\begin{gathered}
\left\{x \in \Omega: d(x, \partial \Omega)>\frac{\delta}{2}\right\} \subset \Omega_{\delta} \\
\subset\left\{x \in \Omega: d(x, \partial \Omega)>\frac{\delta}{3}\right\} .
\end{gathered}
$$

For $\omega \in \mathcal{O}_{\delta}$, we consider the overdetermined Stokes boundary values problem:

$$
\begin{aligned}
-\mu \Delta \mathbf{u}+\nabla p=0 & \text { in } \Omega \backslash \bar{\omega} \\
\operatorname{div} \mathbf{u}=0 & \text { in } \Omega \backslash \bar{\omega} \\
\mathbf{u}=\mathbf{f} & \text { on } \partial \Omega \\
-\mu \partial_{n} \mathbf{u}+p \mathbf{n}=0 & \text { on } \partial \omega \\
-\mu \partial_{n} \mathbf{u}+p \mathbf{n}=\mathbf{g} & \text { on } O
\end{aligned}
$$

where $\mathbf{f} \in \mathbf{H}^{1 / 2}(\partial \Omega)$ such that $\mathbf{f} \neq 0$ and the compatibility condition is fulfilled; that is,

$$
\int_{\partial \Omega} \mathbf{f} . \mathbf{n}=0,
$$

and $\mathbf{g} \in\left[H_{00}^{1 / 2}\right]^{\prime}(O)$ is an admissible boundary measurement. Here $\left[H_{00}^{1 / 2}\right]^{\prime}(O)$ stands for the classical dual space of $H_{00}^{1 / 2}(O)$. The constant $\mu>0$ represents the kinematic viscosity of the fluid, the vectorial function $\mathbf{u}$ represents the velocity of the fluid, and the scalar function $p$ represents the pressure.

Note that we assume that there exists an admissible geometry $\omega^{*} \in \mathcal{O}_{\delta}$ such that (5) has a solution. So that, the geometric inverse problem under consideration reads

Find $\omega \in \mathcal{O}_{\delta}$ and $(\mathbf{u}, p)$

which satisfies the overdetermined system (5).

Our purpose here is to solve the inverse problem of reconstructing $\omega$ using shape optimization techniques. The reader will find all the ingredients for shape differentiation in the papers of Jacques Simon $([5,6])$ and the books of Henrot and Pierre [4] and of Sokolowski and Zolesio [7].

To recover the shape of the inclusion $\omega$, we adopt the usual approach by minimizing a shape functional. We follow the classical technique of optimization; that is, we evaluate an explicit formula of the gradient of the shape functional which can be used in numerical experiments. Many choices of shape functionals are possible. For instance in [2], Badra et al. investigate the problem of the detection of an obstacle in a fluid by boundary measurement, using the least-squares cost functional.

In this paper, following previous works by Caubet et al. in [3], we will solve the inverse problem by using the KohnVogelius cost functional

$$
J_{K V}(\omega)=\frac{1}{2} \int_{\Omega \backslash \bar{\omega}} \mu\left|\nabla\left(\mathbf{u}_{D}-\mathbf{u}_{N}\right)\right|^{2}
$$

where $\left(\mathbf{u}_{D}, p_{D}\right) \in \mathbf{H}^{1}(\Omega \backslash \bar{\omega}) \times \mathbf{L}^{2}(\Omega \backslash \bar{\omega})$ is the unique solution of the Stokes problem with mixed boundary conditions given by

$$
\begin{aligned}
-\mu \Delta \mathbf{u}_{D}+\nabla p_{D}=0 & \text { in } \Omega \backslash \bar{\omega} \\
\operatorname{div} \mathbf{u}_{D}=0 & \text { in } \Omega \backslash \bar{\omega} \\
\mathbf{u}_{D}=\mathbf{f} & \text { on } \partial \Omega \\
-\mu \partial_{n} \mathbf{u}_{D}+p_{D} \mathbf{n}=0 & \text { on } \partial \omega
\end{aligned}
$$

and $\left(\mathbf{u}_{N}, p_{N}\right) \in \mathbf{H}^{1}(\Omega \backslash \bar{\omega}) \times \mathbf{L}^{2}(\Omega \backslash \bar{\omega})$ is the unique solution of the following Stokes problem with Neumann boundary conditions:

$$
\begin{aligned}
-\mu \Delta \mathbf{u}_{N}+\nabla p_{N}=0 & \text { in } \Omega \backslash \bar{\omega} \\
\operatorname{div} \mathbf{u}_{N}=0 & \text { in } \Omega \backslash \bar{\omega} \\
-\mu \partial_{n} \mathbf{u}_{N}+p_{N} \mathbf{n}=\mathbf{g} & \text { on } O \\
\mathbf{u}_{N}=\mathbf{f} & \text { on } \partial \Omega \backslash \bar{O} \\
-\mu \partial_{n} \mathbf{u}_{N}+p_{N} \mathbf{n}=0 & \text { on } \partial \omega .
\end{aligned}
$$

For the results of existence, uniqueness, and regularity of the solutions of the Stokes problem with Neumann boundary conditions, one can refer to [2]. Also the existence result for the mixed boundary conditions is well known. For the sake of clarity, we will recall that result in Appendix.

In order to determine the shape of $\omega$, we try to minimize the Kohn-Vogelius cost functional $J_{K V}(\omega)$ :

$$
\omega^{*}=\underset{\omega \in \mathcal{O}_{\delta}}{\arg \min } J_{K V}(\omega) .
$$

Indeed, if $\omega^{*}$ solves (11) with $J_{K V}\left(\omega^{*}\right)=0$, then this domain $\omega^{*}$ is a solution of the inverse problem (7). Conversely, if $\omega^{*}$. is solution of the inverse problem (7), then $J_{K V}\left(\omega^{*}\right)=0$ and (11) holds.

The Needed Functional Tools. The velocity method is used to define the shape derivatives. For this purpose, we introduce the following space of admissible deformations:

$$
U:=\left\{\theta \in \mathbf{W}^{3, \infty}\left(\mathbb{R}^{d}\right) ; \operatorname{Supp} \theta \subset \overline{\Omega_{\delta}}\right\} .
$$

Then consider for any $\mathbf{V} \in U$ the following application:

$$
\begin{gathered}
\phi: t \in[0, T) \longrightarrow \\
I+t \mathbf{V} \in W^{3, \infty}\left(\mathbb{R}^{d}\right)
\end{gathered}
$$

with $T>0$ being a fixed and small number. Let us notice that, for $t$ small enough, $\phi(t)$ is a diffeomorphism of $\mathbb{R}^{d}$ and $\phi^{\prime}(0)=\mathbf{V}$ vanishes on $\partial \Omega$. Now for $t \in[0, T)$, we define

$$
\begin{aligned}
& \Omega_{t}:=\phi(t)(\Omega), \\
& V_{n}:=\mathbf{V} \cdot \mathbf{n}
\end{aligned}
$$

where $\mathbf{V}$ is a perturbation direction. 
For $u \in \mathbf{H}^{1}(\Omega)$, we recall that the shape derivative is defined by

$$
u^{\prime}=\dot{u}-\nabla u \cdot V
$$

where

$$
\begin{aligned}
& \dot{u}(x)=\lim _{t \rightarrow 0} \frac{u_{t} \circ \phi_{t}(x)-u(x)}{t} \\
& \forall x \in \Omega, u_{t} \in H^{1}\left(\Omega_{t}\right) .
\end{aligned}
$$

For more details on the differentiation with respect to the domain, see [4-7].

\section{Identifiability Result}

This section is devoted to new identifiability result for the mixed case.

Theorem 1 (identifiability result). Let $\Omega \subseteq \mathbb{R}^{d},(d=2$ or $d=$ 3) be a bounded Lipschitz domain and $O$ be a nonempty open subset of $\partial \Omega$. Let $\omega_{0}, \omega_{1} \in \mathcal{O}_{\delta}$ and $\mathbf{f} \in \mathbf{H}^{1 / 2}(\partial \Omega)$ with $\mathbf{f} \neq \mathbf{0}$ satisfying the flux condition $\int_{\partial \Omega} \mathbf{f} \cdot \mathbf{n}=0$. Let $\left(\boldsymbol{u}_{\boldsymbol{j}}, p_{j}\right)$ for $j=0,1$ be a solution of

$$
\begin{aligned}
-\mu \Delta \mathbf{u}_{j}+\nabla p_{j}=\mathbf{0} & \text { in } \Omega \backslash \overline{\omega_{j}} \\
\operatorname{div} \boldsymbol{u}_{j}=0 & \text { in } \Omega \backslash \overline{\omega_{j}}, \\
\boldsymbol{u}_{j}=\boldsymbol{f} & \text { on } \partial \Omega, \\
-\mu \partial_{n_{j}} \mathbf{u}_{j}+p_{j} \mathbf{n}_{j}=\mathbf{0} & \text { on } \partial \omega_{j} .
\end{aligned}
$$

Assume that $\left(\boldsymbol{u}_{\boldsymbol{j}}, p_{j}\right)$ are such that

$$
-\mu \partial_{n_{0}} \mathbf{u}_{0}+p_{0} \mathbf{n}_{0}=-\mu \partial_{n_{1}} \mathbf{u}_{1}+p_{1} \mathbf{n}_{1} \quad \text { on } O .
$$

Then $\omega_{0} \equiv \omega_{1}$.

This result is directly adapted from Theorem 2.2 given in [2] to our problem.

Hence the solution of problem (7) is unique since, for a fixed $f$, the same measure $g$ yields the same geometry $\omega$ in $\mathcal{O}_{\delta}$.

\section{Shape Derivatives of the States}

The following proposition states that the solutions $\left(\mathbf{u}_{D}, p_{D}\right)$ and $\left(\mathbf{u}_{N}, p_{N}\right)$ are differentiable with respect to the domain. Moreover, we obtain a characterization of the shape derivatives of these solutions. This result is based on [2, Proposition 2.5].

Proposition 2 (first-order shape derivatives of the states). Let $\mathbf{V} \in U$ be an admissible deformation. The solutions $\left(\mathbf{u}_{D}, p_{D}\right)$ and $\left(\mathbf{u}_{N}, p_{N}\right)$ are differentiable with respect to the domain and the shape derivatives $\left(\mathbf{u}_{D}^{\prime}, p_{D}^{\prime}\right)$ and $\left(\mathbf{u}_{N}^{\prime}, p_{N}^{\prime}\right)$ belong to $\mathbf{H}^{2}\left(\Omega_{\delta} \backslash\right.$ $\bar{\omega}) \times \mathbf{H}^{1}\left(\Omega_{\delta} \backslash \bar{\omega}\right)$. The couples $\left(\mathbf{u}_{D}^{\prime}, p_{D}^{\prime}\right)$ and $\left(\mathbf{u}_{N}^{\prime}, p_{N}^{\prime}\right) \in \mathbf{H}^{1}(\Omega \backslash$ $\bar{\omega}) \times \mathbf{L}^{2}(\Omega \backslash \bar{\omega})$ are, respectively, the only solutions of the following boundary value problems:

$$
\begin{aligned}
&-\mu \Delta \mathbf{u}_{D}^{\prime}+\nabla p_{D}^{\prime}=0 \text { in } \Omega \backslash \bar{\omega} \\
& \operatorname{div} \mathbf{u}_{D}^{\prime}=0 \text { in } \Omega \backslash \bar{\omega} \\
& \mathbf{u}_{D}^{\prime}=\mathbf{0} \text { on } \partial \Omega \\
&-\mu \partial_{n} \mathbf{u}_{D}^{\prime}+p_{D}^{\prime} \mathbf{n}=\left(\mu \partial_{n n}^{2} \mathbf{u}_{D}-\partial_{n} p_{D} \mathbf{n}\right)(\mathbf{V} . \mathbf{n}) \\
&+p_{D} \nabla_{\Gamma}(\mathbf{V} . \mathbf{n})-\mu \nabla \mathbf{u}_{D} \nabla_{\Gamma}(\mathbf{V} . \mathbf{n}) \\
& \text { on } \partial \omega
\end{aligned}
$$

$$
\begin{aligned}
& \text { and } \\
& -\mu \Delta \mathbf{u}_{N}^{\prime}+\nabla p_{N}^{\prime}=0 \quad \text { in } \Omega \backslash \bar{\omega} \\
& \operatorname{div}\left(\mathbf{u}_{N}^{\prime}\right)=0 \quad \text { in } \Omega \backslash \bar{\omega} \\
& -\mu \partial_{n} \mathbf{u}_{N}^{\prime}+p_{N}^{\prime} \mathbf{n}=0 \text { on } O \\
& \mathbf{u}_{N}^{\prime}=0 \quad \text { on } \partial \Omega \backslash \bar{O} \\
& -\mu \partial_{n} \mathbf{u}_{N}^{\prime}+p_{N}^{\prime} \mathbf{n}=\left(\mu \partial_{n n}^{2} \mathbf{u}_{N}-\partial_{n} p_{N} \mathbf{n}\right)(\mathbf{V} . \mathbf{n}) \\
& +p_{N} \nabla_{\Gamma}(\mathbf{V} . \mathbf{n})-\mu \nabla \mathbf{u}_{N} \nabla_{\Gamma}(\mathbf{V} . \mathbf{n}) \\
& \text { on } \partial \omega \text {. }
\end{aligned}
$$

We aim to compute the gradient of the Kohn-Vogelius functional.

\section{Shape Derivative of the Kohn-Vogelius Cost Functional}

We consider for $\omega \in \mathcal{O}_{\delta}$, the Kohn-Vogelius cost functional

$$
J_{K V}(\omega)=\frac{1}{2} \int_{\Omega \backslash \bar{\omega}} \mu\left|\nabla\left(\mathbf{u}_{D}-\mathbf{u}_{N}\right)\right|^{2} .
$$

To simplify the expressions, we use the following notations:

$$
\begin{aligned}
w & :=\mathbf{u}_{D}-\mathbf{u}_{N}, \\
q & :=p_{D}-p_{N}
\end{aligned}
$$

where $\left(\mathbf{u}_{D}, p_{D}\right)$ solves $(9)$ and $\left(\mathbf{u}_{N}, p_{N}\right)$ solves (10).

Proposition 3 (first-order shape derivative of the functional). For $\mathbf{V} \in U$, the Kohn-Vogelius cost functional $J_{K V}$ is differentiable at $\omega$ in the direction $\mathbf{V}$ with

$$
\begin{aligned}
& D J_{K V}(\omega) . \mathbf{V}=\frac{1}{2} \int_{\partial \omega} \mu|\nabla w|^{2} \mathbf{V}_{n} \\
& \quad+\int_{\partial \omega}\left[\left(\mu \partial_{n n}^{2} \mathbf{u}_{N}-\partial_{n} p_{N} \mathbf{n}\right)(\mathbf{V} \cdot \mathbf{n})+p_{N} \nabla_{\Gamma}(\mathbf{V} . \mathbf{n})\right. \\
& \left.\quad-\mu \nabla \mathbf{u}_{N} \nabla_{\Gamma}(\mathbf{V} \cdot \mathbf{n})\right] . w
\end{aligned}
$$

where $(w, q)$ is defined by (22). 
Proof. From Hadamard's formula (see [4, Theorem 5.2.2]), we have

$$
\begin{aligned}
& D J_{K V}(\omega) \cdot \mathbf{V} \\
& \quad=\int_{\Omega \backslash \bar{\omega}}\left[\mu \nabla w: \nabla w^{\prime}+\frac{1}{2} \mu \operatorname{div}\left(|\nabla w|^{2} \mathbf{V}\right)\right] \\
& \quad=\int_{\Omega \backslash \bar{\omega}} \mu \nabla w: \nabla w^{\prime}+\frac{1}{2} \int_{\partial(\Omega \backslash \bar{\omega})} \mu|\nabla w|^{2} \mathbf{V}_{n} \\
& \quad=\int_{\Omega \backslash \bar{\omega}} \mu \nabla w: \nabla\left(\mathbf{u}_{D}^{\prime}-\mathbf{u}_{N}^{\prime}\right)+\frac{1}{2} \int_{\partial \omega} \mu|\nabla w|^{2} \mathbf{V}_{n}
\end{aligned}
$$

because $\mathbf{V}=0$ on $\partial \Omega$. As

$$
\begin{aligned}
& \int_{\Omega \backslash \bar{\omega}} \mu \nabla w: \nabla\left(\mathbf{u}_{D}^{\prime}-\mathbf{u}_{N}^{\prime}\right) \\
& =\int_{\Omega \backslash \bar{\omega}} \mu \nabla w: \nabla \mathbf{u}_{D}^{\prime}-\int_{\Omega \backslash \bar{\omega}} \mu \nabla w: \nabla \mathbf{u}_{N}^{\prime}
\end{aligned}
$$

we apply Green Formula for $\int_{\Omega \backslash \bar{\omega}} \mu \nabla w: \nabla \mathbf{u}_{D}^{\prime}$ :

$$
\begin{aligned}
\int_{\Omega \backslash \bar{\omega}} \mu \nabla w: \nabla \mathbf{u}_{D}^{\prime}= & -\int_{\Omega \backslash \bar{\omega}} \mu \Delta w \cdot \mathbf{u}_{D}^{\prime} \\
& +\int_{\partial(\Omega \backslash \bar{\omega})} \mu \partial_{n} w \cdot \mathbf{u}_{D}^{\prime} \\
= & -\int_{\Omega \backslash \bar{\omega}} \nabla q \cdot \mathbf{u}_{D}^{\prime}+\int_{\partial(\Omega \backslash \bar{\omega})} \mu \partial_{n} w \cdot \mathbf{u}_{D}^{\prime} \\
= & \int_{\Omega \backslash \bar{\omega}} q \operatorname{div} \mathbf{u}_{D}^{\prime}-\int_{\partial(\Omega \backslash \bar{\omega})} q \mathbf{u}_{D}^{\prime} \mathbf{n} \\
& +\int_{\partial(\Omega \backslash \bar{\omega})} \mu \partial_{n} w \cdot \mathbf{u}_{D}^{\prime} \\
= & \int_{\partial(\Omega \backslash \bar{\omega})}\left[\mu \partial_{n} w-q \mathbf{n}\right] \cdot \mathbf{u}_{D}^{\prime} \\
= & \int_{\partial \Omega}\left[\mu \partial_{n} w-q \mathbf{n}\right] \cdot \mathbf{u}_{D}^{\prime} \\
& +\int_{\partial \omega}\left[\mu \partial_{n} w-q \mathbf{n}\right] \cdot \mathbf{u}_{D}^{\prime} .
\end{aligned}
$$

Since $\operatorname{div} \mathbf{u}_{D}^{\prime}=0$ in $\Omega \backslash \bar{\omega}$ with $\mathbf{u}_{D}^{\prime}=0$ on $\partial \Omega$ and $-\mu \partial_{n} \mathbf{u}_{N}+p_{N} \mathbf{n}=-\mu \partial_{n} \mathbf{u}_{D}+p_{D} \mathbf{n}=0$ on $\partial \omega$,

$$
\int_{\Omega \backslash \bar{\omega}} \mu \nabla\left(\mathbf{u}_{D}-\mathbf{u}_{N}\right): \nabla \mathbf{u}_{D}^{\prime}=0
$$

Apply now Green Formula for $\int_{\Omega \backslash \bar{\omega}} \mu \nabla w: \nabla \mathbf{u}_{N}^{\prime}$ to get

$$
\begin{gathered}
\int_{\Omega \backslash \bar{\omega}} \mu \nabla w: \nabla \mathbf{u}_{N}^{\prime}=-\int_{\Omega \backslash \bar{\omega}} \mu \Delta \mathbf{u}_{N}^{\prime} \cdot w+\int_{\partial(\Omega \backslash \bar{\omega})} \mu \partial_{n} \mathbf{u}_{N}^{\prime} \cdot \\
w=-\int_{\Omega \backslash \bar{\omega}} \nabla p_{N}^{\prime} \cdot w+\int_{\partial(\Omega \backslash \bar{\omega})} \mu \partial_{n} \mathbf{u}_{N}^{\prime} \cdot w=\int_{\Omega \backslash \bar{\omega}} p_{N}^{\prime}
\end{gathered}
$$

$$
\begin{aligned}
& \cdot \operatorname{div} w-\int_{\partial(\Omega \backslash \bar{\omega})} p_{N}^{\prime} w \cdot \mathbf{n}+\int_{\partial(\Omega \backslash \bar{\omega})} \mu \partial_{n} \mathbf{u}_{N}^{\prime} \cdot w \\
& =\int_{\partial(\Omega \backslash \bar{\omega})}\left(\mu \partial_{n} \mathbf{u}_{N}^{\prime}-p_{N}^{\prime} \mathbf{n}\right) \cdot w=\int_{\partial \Omega}\left(\mu \partial_{n} \mathbf{u}_{N}^{\prime}\right. \\
& \left.-p_{N}^{\prime} \mathbf{n}\right) \cdot w+\int_{\partial \omega}\left(\mu \partial_{n} \mathbf{u}_{N}^{\prime}-p_{N}^{\prime} \mathbf{n}\right) \cdot w \\
& =-\int_{\partial \omega}\left[\left(\mu \partial_{n n}^{2} \mathbf{u}_{N}-\partial_{n} p_{N} \mathbf{n}\right)(\mathbf{V} \cdot \mathbf{n})+p_{N} \nabla_{\Gamma}(\mathbf{V} \cdot \mathbf{n})\right. \\
& \left.-\mu \nabla \mathbf{u}_{N} \nabla_{\Gamma}(\mathbf{V} \cdot \mathbf{n})\right] \cdot w .
\end{aligned}
$$

Since $\operatorname{div} w=0$ in $\Omega \backslash \bar{\omega}$ with $-\mu \partial_{n} \mathbf{u}_{N}^{\prime}+p_{N}^{\prime} \mathbf{n}=0$ on $\partial \Omega$ and from (20)

$$
\begin{aligned}
-\mu \partial_{n} \mathbf{u}_{N}^{\prime}+p_{N}^{\prime} \mathbf{n}= & \left(\mu \partial_{n n}^{2} \mathbf{u}_{N}-\partial_{n} p_{N} \mathbf{n}\right)(\mathbf{V . n}) \\
& +p_{N} \nabla_{\Gamma}(\mathbf{V . n})-\mu \nabla \mathbf{u}_{N} \nabla_{\Gamma}(\mathbf{V . n})
\end{aligned}
$$

on $\partial \omega$

thus we get

$$
\begin{aligned}
& \int_{\Omega \backslash \bar{\omega}} \mu \nabla w: \nabla \mathbf{u}_{N}^{\prime}=-\int_{\partial \omega}\left[\left(\mu \partial_{n n}^{2} \mathbf{u}_{N}-\partial_{n} p_{N} \mathbf{n}\right)(\mathbf{V} . \mathbf{n})\right. \\
& \left.+p_{N} \nabla_{\Gamma}(\mathbf{V} . \mathbf{n})-\mu \nabla \mathbf{u}_{N} \nabla_{\Gamma}(\mathbf{V} . \mathbf{n})\right] . w .
\end{aligned}
$$

From (27)-(30), we get

$$
\begin{gathered}
\int_{\Omega \backslash \bar{\omega}} \mu \nabla w: \nabla w^{\prime}=\int_{\partial \omega}\left[\left(\mu \partial_{n n}^{2} \mathbf{u}_{N}-\partial_{n} p_{N} \mathbf{n}\right)(\mathbf{V . n})\right. \\
\left.+p_{N} \nabla_{\Gamma}(\mathbf{V} . \mathbf{n})-\mu \nabla \mathbf{u}_{N} \nabla_{\Gamma}(\mathbf{V} . \mathbf{n})\right] . w
\end{gathered}
$$

Hence the first-order shape derivative of the functional is

$$
\begin{aligned}
& D J_{K V}(\omega) \cdot \mathbf{V}=\frac{1}{2} \int_{\partial \omega} \mu|\nabla w|^{2} \mathbf{V}_{n} \\
& \quad+\int_{\partial \omega}\left[\left(\mu \partial_{n n}^{2} \mathbf{u}_{N}-\partial_{n} p_{N} \mathbf{n}\right)(\mathbf{V} \cdot \mathbf{n})+p_{N} \nabla_{\Gamma}(\mathbf{V} \cdot \mathbf{n})\right. \\
& \left.\quad-\mu \nabla \mathbf{u}_{N} \nabla_{\Gamma}(\mathbf{V} . \mathbf{n})\right] . w .
\end{aligned}
$$

To recover the shape of the inclusion $\omega$, we adopt the usual approach by minimizing a shape functional. We follow the classical technique of optimization; that is, we evaluate an explicit formula of the gradient of the shape functional which can be used in numerical experiments. The gradient is computed component by component using its characterization (see Proposition 3, formula (23). The optimization method used for the numerical simulations is the classical gradient algorithm which is the descent method: For a given initial shape $\omega_{0}$, we can compute the following iteration by the algorithm $\omega_{i+1}=\omega_{i}-\alpha_{i} \nabla J_{K V}\left(\omega_{i}\right)$ where $\alpha_{i}$ is a satisfying step length, until obtaining the stopped criterion. For more details see [3]. 


\section{Conclusion}

We solved our inverse problem using shape optimization methods to detect an inclusion immersed in a fluid. We use here the functional Kohn-Vogelius; we compute the first shape derivative of this functional which can be used in numerical experiments.

\section{Appendix}

\section{Result on the Stokes Problem with Mixed Conditions}

Define

$$
\begin{gathered}
\mathbf{S}_{O}(\Omega \backslash \bar{\omega}):=\left\{\boldsymbol{u} \in \boldsymbol{H}^{1}(\Omega \backslash \bar{\omega}) ; \operatorname{div} \mathbf{u}=0 \text { in } \Omega \backslash \bar{\omega}, \mathbf{u}\right. \\
\left.=0 \text { on } \partial \Omega \backslash \bar{O} \text { and }-\mu \partial_{\mathbf{n}} \boldsymbol{u}+p \mathbf{n}=\mathbf{0} \text { on } \partial \omega\right\}
\end{gathered}
$$

and denote, respectively, by $\langle\cdot, \cdot\rangle_{\Omega \backslash \bar{\omega}}$ and $\langle\cdot, \cdot\rangle_{O}$ the duality product between $\left[\mathbf{H}^{1}(\Omega \backslash \bar{\omega})\right]^{\prime}$ and $\mathbf{H}^{1}(\Omega \backslash \bar{\omega})$ and the duality product between $\mathbf{H}^{-1 / 2}(O)$ and $\mathbf{H}^{1 / 2}(O)$.

Theorem A.1 (existence and uniqueness of the solution). Let $\Omega$ be a bounded Lipschitz open set of $\mathbb{R}^{d}\left(d \in \mathbb{N}^{*}\right)$ and let $\omega \subset \subset$ $\Omega$ be a Lipschitz open subset of $\Omega$ such that $\Omega \backslash \bar{\omega}$ is connected. Let $O$ be an open subset of the exterior boundary $\partial \Omega$ and $\mu>0$. Let $\boldsymbol{f} \in \mathbf{L}^{2}(\Omega \backslash \bar{\omega}), \boldsymbol{h}_{O} \in \mathbf{H}^{-1 / 2}(O), \mathbf{h}_{\text {ext }} \in \mathbf{H}^{1 / 2}(\partial \Omega \backslash \bar{O})$, and $\mathbf{h}_{\text {int }} \in \mathbf{H}^{-1 / 2}(\partial \omega)$. Then, the problem.

$$
\begin{aligned}
& -\mu \Delta \boldsymbol{u}+\nabla p=\mathbf{f} \quad \text { in } \Omega \backslash \bar{\omega} \\
& \operatorname{div} \mathbf{u}=0 \quad \text { in } \Omega \backslash \bar{\omega} \\
& -\mu \partial_{\mathbf{n}} \boldsymbol{u}+p \mathbf{n}=\boldsymbol{h}_{O} \quad \text { on } O \\
& \mathbf{u}=\mathbf{h}_{\text {ext }} \quad \text { on } \partial \Omega \backslash \bar{O} \\
& -\mu \partial_{\mathbf{n}} \mathbf{u}+p \mathbf{n}=\mathbf{h}_{\text {int }} \quad \text { on } \partial \omega
\end{aligned}
$$

admits a unique solution $(\boldsymbol{u}, p) \in \mathbf{H}^{1}(\Omega \backslash \bar{\omega}) \times \mathbf{L}^{2}(\Omega \backslash \bar{\omega})$.

Proof. According to [8, Lemma 3.3], consider $\mathbf{H} \in \mathbf{H}^{1}(\Omega \backslash \bar{\omega})$ such that $\operatorname{div} \mathbf{H}=0,-\mu \partial_{\mathbf{n}} \mathbf{H}=\mathbf{h}_{\text {int }}$ on $\partial \omega$, and $\mathbf{H}=\mathbf{h}_{\text {ext }}$ on $\partial \Omega \backslash \bar{O}$ such that $\int_{\partial \Omega \cup \partial \omega} \mathbf{H . n}=0$. Then the couple $(\mathbf{U}:=$ $\boldsymbol{u}-\mathbf{H}, p) \in \mathbf{H}^{1}(\Omega \backslash \bar{\omega}) \times \mathrm{L}^{2}(\Omega \backslash \bar{\omega})$ satisfies

$$
\begin{aligned}
&-\mu \Delta \mathbf{U}+\nabla p=\boldsymbol{f}+\mu \Delta \mathbf{H} \quad \text { in } \Omega \backslash \bar{\omega} \\
& \operatorname{div} \mathbf{U}=0 \quad \text { in } \Omega \backslash \bar{\omega} \\
&-\mu \partial_{\mathbf{n}} \mathbf{U}+p \mathbf{n}=\mathbf{h}_{O}+\mu \partial_{\mathbf{n}} \mathbf{H} \quad \text { on } O \\
& \mathbf{U}=\mathbf{0} \quad \text { on } \partial \Omega \backslash \bar{O} \\
&-\mu \partial_{\mathbf{n}} \mathbf{U}+p \mathbf{n}=\mathbf{0} \text { on } \partial \omega .
\end{aligned}
$$

From the first equation we obtain, for $\mathbf{v} \in \mathbf{S}_{\mathrm{O}}(\Omega \backslash \bar{\omega})$,

$$
\int_{\Omega \backslash \bar{\omega}}(-\mu \Delta \mathbf{U}+\nabla p) \mathbf{v}=\int_{\Omega \backslash \bar{\omega}}(f+\mu \Delta \mathbf{H}) \mathbf{v}
$$

Apply now Green Formula to get

$$
\begin{aligned}
& \mu \int_{\Omega \backslash \bar{\omega}} \nabla \mathbf{U}: \nabla \mathbf{v}-\mu \int_{\partial(\Omega \backslash \bar{\omega})} \partial_{\mathbf{n}} \mathbf{U} \cdot \mathbf{v}+\int_{\Omega \backslash \bar{\omega}} \nabla p \cdot \mathbf{v} \\
& \quad=\langle\mathbf{f}, \mathbf{v}\rangle_{\Omega \backslash \bar{\omega}}-\mu \int_{\Omega \backslash \bar{\omega}} \nabla \mathbf{H}: \nabla \mathbf{v}+\mu \int_{\partial(\Omega \backslash \bar{\omega})} \partial_{\mathbf{n}} \mathbf{H} . \mathbf{v}
\end{aligned}
$$

Since we have

$$
\int_{\Omega \mid \bar{\omega}} \nabla p \cdot \mathbf{v}=-\int_{\Omega \mid \bar{\omega}} p \cdot \operatorname{div} \mathbf{v}+\int_{\partial(\Omega \mid \bar{\omega})} p n \cdot \mathbf{v}
$$

then we obtain

$$
\begin{aligned}
& \mu \int_{\Omega \backslash \bar{\omega}} \nabla \mathbf{U}: \nabla \mathbf{v}+\int_{\partial(\Omega \backslash \bar{\omega})}\left(-\mu \partial_{\mathbf{n}} \mathbf{U}+p n\right) \cdot \mathbf{v} \\
& \quad=\langle\mathbf{f}, \mathbf{v}\rangle_{\Omega \backslash \bar{\omega}}-\mu \int_{\Omega \backslash \bar{\omega}} \nabla \mathbf{H}: \nabla \mathbf{v}+\mu \int_{\partial(\Omega \backslash \bar{\omega})} \partial_{\mathbf{n}} \mathbf{H . v}
\end{aligned}
$$

From the conditions on the boundary we get

$$
\begin{aligned}
\mu \int_{\Omega \backslash \bar{\omega}} \nabla \mathbf{U}: \nabla \mathbf{v}= & \langle\mathbf{f}, \mathbf{v}\rangle_{\Omega \backslash \bar{\omega}}-\mu \int_{\Omega \backslash \bar{\omega}} \nabla \mathbf{H}: \nabla \mathbf{v} \\
& -\left\langle\mathbf{h}_{O}+\mu \partial_{\mathbf{n}} \mathbf{H}, \mathbf{v}\right\rangle_{O}
\end{aligned}
$$

From Lax-Milgram's Theorem, there exists a unique $\mathbf{U} \in$ $\mathbf{S}_{O}(\Omega \backslash \bar{\omega})$ such that, for all $\mathbf{v} \in \mathbf{S}_{O}(\Omega \backslash \bar{\omega})$, one has

$$
\begin{aligned}
\mu \int_{\Omega \backslash \bar{\omega}} \nabla \mathbf{U}: \nabla \mathbf{v}= & \langle\mathbf{f}, \mathbf{v}\rangle_{\Omega \backslash \bar{\omega}}-\mu \int_{\Omega \backslash \bar{\omega}} \nabla \mathbf{H}: \nabla \mathbf{v} \\
& -\left\langle\mathbf{h}_{O}+\mu \partial_{\mathbf{n}} \mathbf{H}, \mathbf{v}\right\rangle_{O}
\end{aligned}
$$

In particular (A.9) is true for all $\mathbf{v} \in \mathbf{S}_{O}(\Omega \backslash \bar{\omega}) \cap \mathbf{H}_{0}^{1}(\Omega \backslash$ $\bar{\omega})$. Then using De Rham's theorem (see [9]), there exists $p \in$ $\mathbf{L}^{2}(\Omega \backslash \bar{\omega})$, up to an additive constant, such that, for all $\mathbf{v} \in$ $\mathbf{H}_{0}^{1}(\Omega \backslash \bar{\omega})$,

$$
\begin{aligned}
\mu \int_{\Omega \backslash \bar{\omega}} \nabla \mathbf{U}: \nabla \mathbf{v}-\int_{\Omega \backslash \bar{\omega}} p \operatorname{div} \mathbf{v} \\
=\left\langle\mathbf{f}_{\mid \mathbf{H}_{0}^{1}(\Omega \backslash \bar{\omega})}, \mathbf{v}\right\rangle_{\mathbf{H}^{-1}(\Omega \mid \bar{\omega}), \mathbf{H}_{0}^{1}(\Omega \backslash \bar{\omega})} \\
\quad-\mu \int_{\Omega \backslash \bar{\omega}} \nabla \mathbf{H}: \nabla \mathbf{v} .
\end{aligned}
$$

Using [8, Lemma 3.3] (or [10, Théorème 3.2]), we define $\varphi_{N} \epsilon$ $\mathbf{H}^{1}(\Omega \backslash \bar{\omega})$ such that $\operatorname{div} \boldsymbol{\varphi}_{N}=1$ in $\Omega \backslash \bar{\omega}, \boldsymbol{\varphi}_{N}=\mathbf{0}$ on $\partial \Omega \backslash \bar{O}$, and $\boldsymbol{\varphi}_{N}=\mathbf{0}$ on $\partial \omega$ with $\int_{O} \boldsymbol{\varphi}_{N} \cdot \mathbf{n} \neq 0$. Let $\mathbf{v} \in \mathbf{H}^{1}(\Omega \backslash \bar{\omega})$ such that $\mathbf{v}=\mathbf{0}$ on $\partial \Omega \backslash \bar{O}$ and $-\mu \partial_{\mathbf{n}} \mathbf{v}+p \mathbf{n}=\mathbf{0}$ on $\partial \omega$ and define

$$
c_{b}(\mathbf{v})=\frac{1}{\int_{\partial(\Omega \backslash \bar{\omega})} \boldsymbol{\varphi}_{N} \cdot \mathbf{n}} \int_{\partial(\Omega \backslash \bar{\omega})} \mathbf{v} \cdot \mathbf{n} .
$$

From [8, Lemma 3.3] (see also [10, Théorème 3.2]), we define $\boldsymbol{v}_{2} \in S_{O}(\Omega \backslash \bar{\omega})$ such that $\boldsymbol{v}=\boldsymbol{v}_{1}+\boldsymbol{v}_{2}+c_{b}(\boldsymbol{v}) \boldsymbol{\varphi}_{N}$, where $\boldsymbol{v}_{1} \epsilon$ $\mathbf{H}_{0}^{1}(\Omega \backslash \bar{\omega})$ satisfies the following equality:

$$
\operatorname{div} \boldsymbol{v}_{1}=\operatorname{div}\left(\boldsymbol{v}-c_{b}(\boldsymbol{v}) \boldsymbol{\varphi}_{N}\right) .
$$


Then, using (A.9) and (A.10), it yields

$$
\begin{aligned}
\mu \int_{\Omega \backslash \bar{\omega}} & \nabla \mathbf{U}: \nabla \mathbf{v}-\int_{\Omega \backslash \bar{\omega}} p \operatorname{div} \mathbf{v} \\
= & \langle\mathbf{f}, \mathbf{v}\rangle_{\Omega \backslash \bar{\omega}}-\mu \int_{\Omega \backslash \bar{\omega}} \nabla \mathbf{H}: \nabla \mathbf{v} \\
& -\left\langle\mathbf{h}_{O}+\mu \partial_{\mathbf{n}} \mathbf{H}, \mathbf{v}\right\rangle_{O} \\
& +\int_{\Omega \backslash \bar{\omega}} \mu \nabla \mathbf{U}: \nabla\left(c_{b}(\mathbf{v}) \boldsymbol{\varphi}_{N}\right) \\
& -\int_{\Omega \backslash \bar{\omega}} p \operatorname{div}\left(c_{b}(\mathbf{v}) \boldsymbol{\varphi}_{N}\right)-\left\langle\mathbf{f}, c_{b}(\mathbf{v}) \boldsymbol{\varphi}_{N}\right\rangle_{\Omega \backslash \bar{\omega}} \\
& +\mu \int_{\Omega \backslash \bar{\omega}} \nabla \mathbf{H}: \nabla\left(c_{b}(\mathbf{v}) \boldsymbol{\varphi}_{N}\right) \\
& +\left\langle\mathbf{h}_{\mathbf{O}}+\mu \partial_{\mathbf{n}} \mathbf{H}, c_{b}(\mathbf{v}) \boldsymbol{\varphi}_{N}\right\rangle_{O} .
\end{aligned}
$$

Choose the additive constant for $p$ such that

$$
\begin{aligned}
\int_{\Omega \backslash \bar{\omega}} p= & \mu \int_{\Omega \backslash \bar{\omega}} \nabla \mathbf{U}: \nabla \boldsymbol{\varphi}_{N}-\left\langle\mathbf{f}, c_{b}(\mathbf{v}) \boldsymbol{\varphi}_{N}\right\rangle_{\Omega \backslash \bar{\omega}} \\
& +\mu \int_{\Omega \backslash \bar{\omega}} \nabla \mathbf{H}: \nabla \boldsymbol{\varphi}_{N} \\
& +\left\langle\mathbf{h}_{O}+\mu \partial_{\mathbf{n}} \mathbf{H}, c_{b}(\mathbf{v}) \boldsymbol{\varphi}_{N}\right\rangle_{O} .
\end{aligned}
$$

Hence, we prove that there exists a unique pair $(\mathbf{U}, p) \epsilon$ $\mathbf{S}_{\mathrm{O}}(\Omega \backslash \bar{\omega}) \times \mathbf{L}^{2}(\Omega \backslash \bar{\omega})$ such that, for all $\mathbf{v} \in \mathbf{H}^{1}(\Omega \backslash \bar{\omega})$ with $\mathbf{v}=\mathbf{0}$ on $\partial \Omega \backslash \bar{O}$ and $-\mu \partial_{\mathbf{n}} \mathbf{v}+p \mathbf{n}=\mathbf{0}$ on $\partial \omega$,

$$
\begin{gathered}
\int_{\Omega \backslash \bar{\omega}} \mu \nabla \mathbf{U}: \nabla \mathbf{v}-\int_{\Omega \backslash \bar{\omega}} p \operatorname{div} \mathbf{v}=\langle\mathbf{f}, \mathbf{v}\rangle_{\Omega \backslash \bar{\omega}} \\
-\mu \int_{\Omega \backslash \bar{\omega}} \nabla \mathbf{H}: \nabla \mathbf{v}-\left\langle\mathbf{h}_{\mathbf{O}}+\mu \partial_{\mathbf{n}} \mathbf{H}, \mathbf{v}\right\rangle_{O}
\end{gathered}
$$

which complete the proof.

\section{Data Availability}

No data were used to support this study.

\section{Conflicts of Interest}

The author declares that she has no conflicts of interest.

\section{References}

[1] C. Alvarez, C. Conca, L. Friz, O. Kavian, and J. H. Ortega, "Identification of immersed obstacles via boundary measurements," Inverse Problems, vol. 21, no. 5, pp. 1531-1552, 2005.

[2] M. Badra, F. Caubet, and M. Dambrine, "Detecting an obstacle immersed in a fluid by shape optimization methods," Mathematical Models and Methods in Applied Sciences, vol. 21, no. 10, pp. 2069-2101, 2011.

[3] F. Caubet, M. Dambrine, D. Kateb, and C. Z. Timimoun, "A Kohn-Vogelius formulation to detect an obstacle immersed in a fluid," Inverse Problems and Imaging, vol. 7, no. 1, pp. 123-157, 2013.
[4] A. Henrot and M. Pierre, Variation et Optimisation De Formes, vol. 48 of Mathématiques et Applications, Springer, Berlin, Germany, 2005.

[5] J. Simon, "Differentiation with respect to the domain in boundary value problems," Numerical Functional Analysis and Optimization, vol. 2, no. 7-8, pp. 649-687 (1981), 1980.

[6] J. Simon, "Second variation for domain optimization problems," in Controland Estimation of Distributed Parameter Systems, F. Kappel, K. Kunisch, and W. Schappacher, Eds., vol. 91 of International Series of Numerical Mathematics, pp. 361-378, Birkhäuser, 1992.

[7] J. Sokolowski and J.-P. Zolesio, Introduction to Shape Optimization: Shape Sensitivity Analysis, vol. 16 of Series in computational Mathematics, Springer, Berlin, Germany, 1992.

[8] C. Amrouche and V. Girault, "Decomposition of vector spaces and application to the Stokes problem in arbitrary dimension," Czechoslovak Mathematical Journal, vol. 44(119), no. 1, pp. 109140, 1994

[9] C. Amrouche and V. Girault, "Problèmes généralis és de Stokes," Portugaliae Mathematica, vol. 49, no. 4, pp. 463-503, 1992.

[10] G. P. Galdi, An Introduction to the Mathematical Theory of the Navier-Stokes Equations, vol. 38 of New York Linearized steady problems, Springer, 1994. 


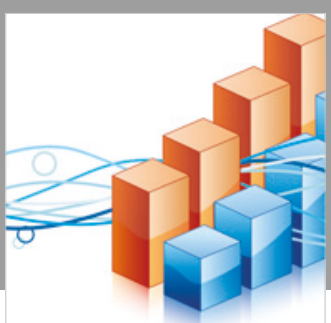

Advances in

Operations Research

\section{-n-m}
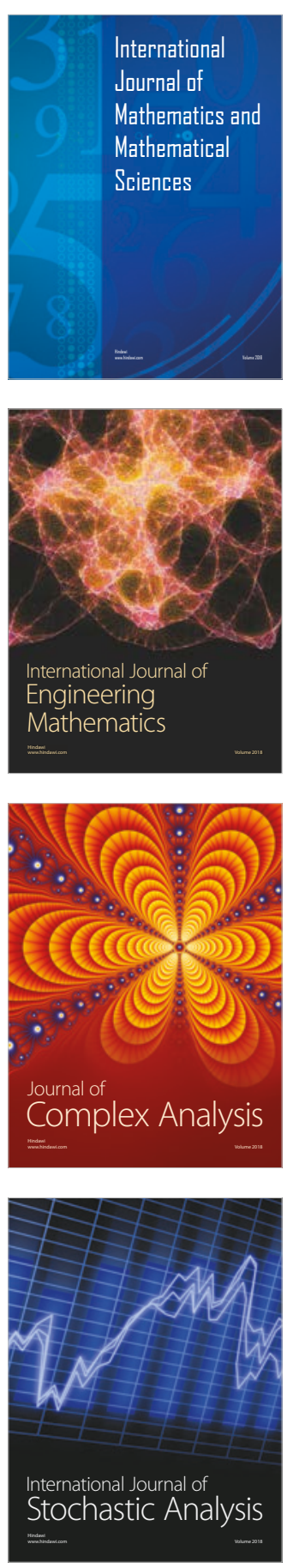
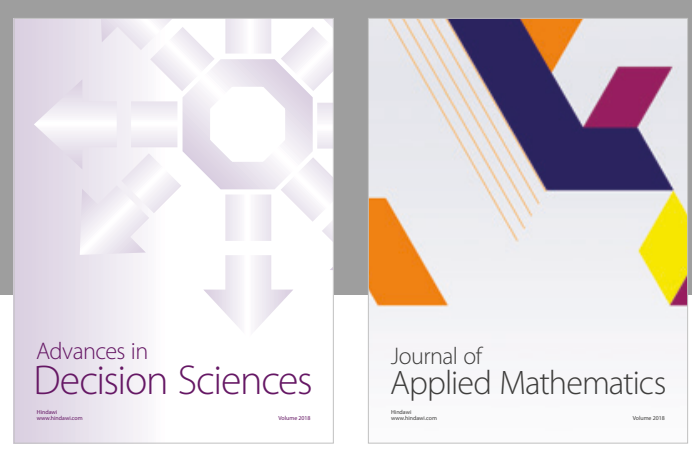

Journal of

Applied Mathematics
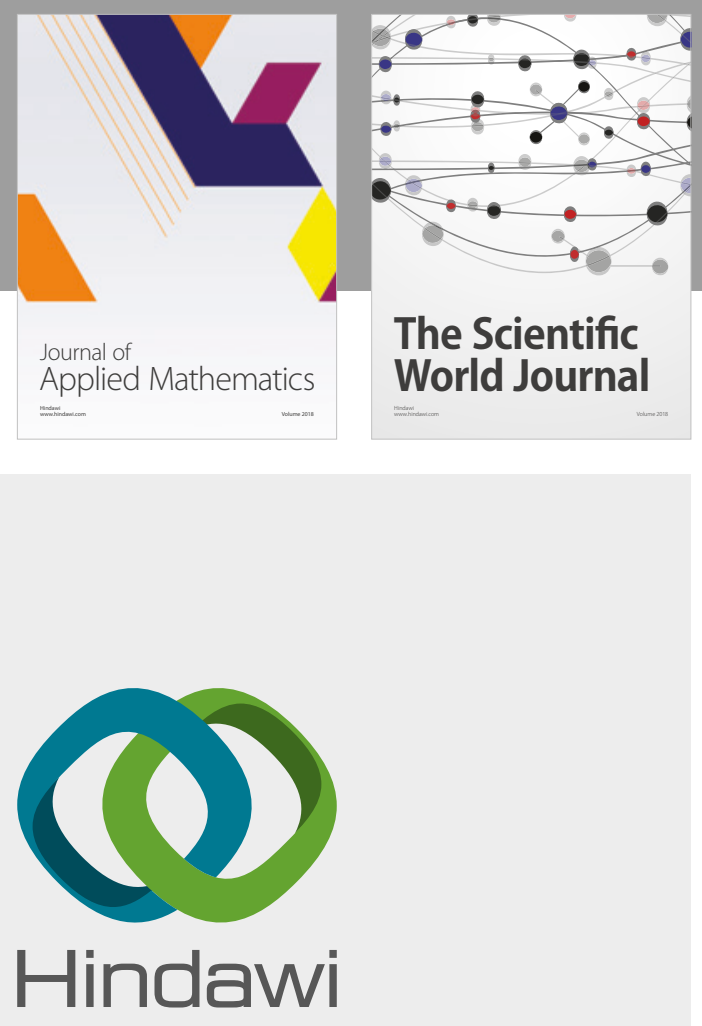

Submit your manuscripts at

www.hindawi.com

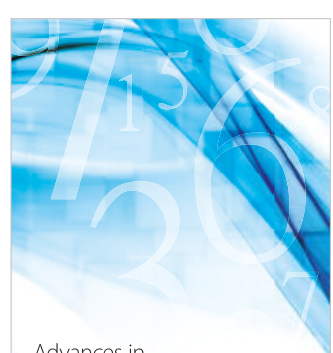

Advances in
Numerical Analysis
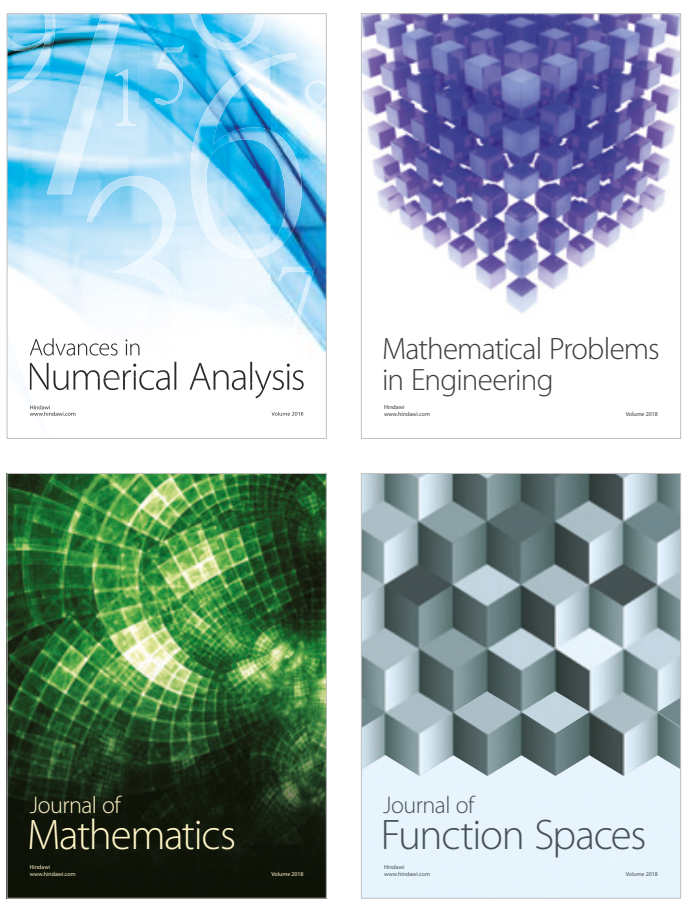

Mathematical Problems in Engineering

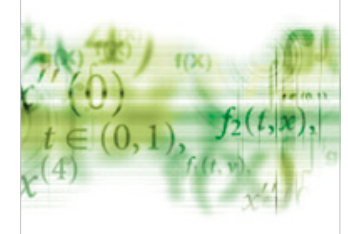

International Journal of

Differential Equations

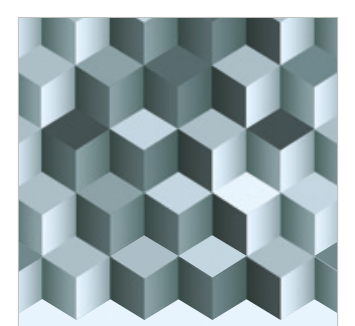

Journal of

Function Spaces

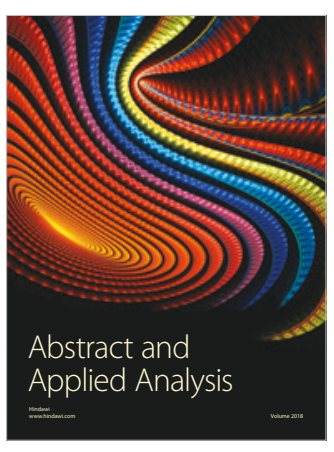

The Scientific

World Journal

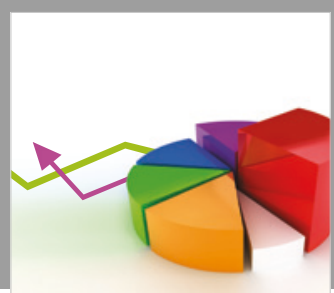

Journal of

Probability and Statistics
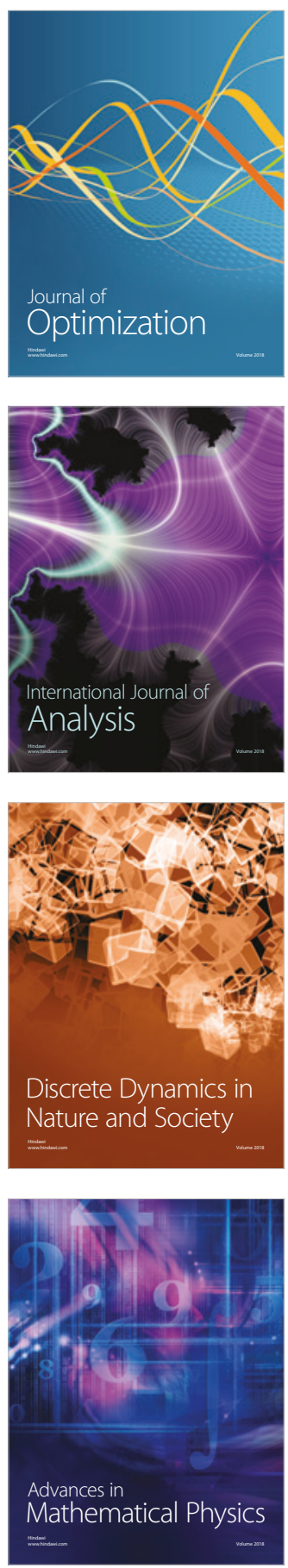